\title{
Cross-Term Suppression in Time Order Distribution for AWGN Signal
}

\author{
WAQAS MAHMOOD*, NABEEL ALI KHAN**, NOOR MUHAMMAD MEMON***, AND FAYYAZ \\ AHMED CHAUDHRY ****
}

RECEIVED ON 30.11.2015 ACCEPTED ON 11.05.2016

\begin{abstract}
A technique of cross-term suppression in WD (Wigner Distribution) for a multi-component signal that is embedded WGN (White Gaussian Noise) is proposed. In this technique, an optimized algorithm is developed for time-varying noisy signal and a CAD (Computer Aided Design) simulator is designed for Numerical simulations of synthetic signal. In proposed technique, signal components are localized in t$f$ (time frequency) plane by STFT (Short Time Fourier Transform). Rectified STFT is computed and Spectral Kurtosis is used to separate a signal components from noise in t-f plane. The t-f plane is segmented and then signal components are filtered out by FFT (Fractional Fourier Transform). Finally, WD (free of cross terms) of isolated signal component is computed to obtain high resolution in t-f plane.
\end{abstract}

Key Words: Short Time Fourier Transform, Wigner Distribution, Time Frequency, Fractional Fourier Transform.

\section{INTRODUCTION}

T

he signals whose spectral contents vary w.r.t time are called Non-stationary signals. These signals cannot be well examined separately in time or frequency domain [1]. In time domain representation, desired information is hidden from analyzer in the form of spectral contents [2-3]. On the other hand, frequency domain representation fails to describe how much spectral contents vary with respect to the time. Therefore, joint time frequency representation is studied for nonstationary signal in 2D (Two Dimensional) plane which gives ideal energy concentration and spectral information along time [3].
Joint t-f representation is a powerful tool for analyzing time varying signal such as radar, biomedical, seismic and sonar signals etc. [4].Time frequency representation can be further divided into two categories i.e. Linear and Quadratic t-f representation. Linear $t-f$ representations are Short STFT, GT (Gabor Transform), and WT (Wavelet Transform), etc. while a Quadratic t-f representation (called bilinear) are WD and GWT (Gabor Wigner Transform), etc. Linear t-f representation provides low resolution for auto-components and no cross-terms for multi-component signal. Quadratic t-f representation provides high resolution for auto-components and cross-

\footnotetext{
* University of Engineering \& Technology, Taxila.

** $\quad$ Foundation University, Islamabad.

*** Quaid-e-Awam University of Engineering \& Technology, Nawabshah.

**** COMSATS Institute of Information \& Technology, Wah Campus.
}

Mehran University Research Journal of Engineering \& Technology, Volume 36, No. 2, April, 2017 [p-ISSN: 0254-7821, e-ISSN: 2413-7219] 
terms for multi-component signal [3-5]. Time Frequency Representation are often compared in term of high readability performance, ability of suppressing unwanted interference term called cross-terms and mathematical formulation [6].

The most popular quadratic $t-f$ representation is WD due to its attractive mathematical properties. WD offers high readability and optimal energy concentration in t-f plane [7]. On the other hand, it has severely severed crossterms due to its quadratic nature. These cross-terms arises an unwanted oscillation in multi-component signal which affect its practical significance. There are several different ways to formulate a free cross-terms t-f representation, but either its computational cost is high or inefficient in term of high resolution [3].

In Cohen's distribution, WD is convolved with 2D kernel function. Cross-term in WD is suppressed by convolution, however, an auto-component lost high resolution as a result of convolution. All $\mathrm{t}$-f representation that is based upon kernel function shows comparison between cross-term suppression and auto-component resolution [1].

FRFT that is based on signal synthesis eliminates crossterms in WD to realm the quality of auto-component was proposed by [8-10]. Moreover, this technique gave optimum energy concentration even when an autocomponent overlaid by cross-terms. Due to iterative nature its computational cost is high which limit its scope for parallel implementation [3].

GWT is a combination of GT and WD. Due to linear property of GT auto-components of signals avoid the presence of cross-terms, which leads to a considerable amount of resolution and energy concentration [3-11]. However, if an auto-component overlap the cross term, then this technique fails to give optimum result.
Another cross term suppression technique was proposed by Khan et. al. [3]. This method is based on Image processing and FFT technique. In this technique SVM (Support Vector Machine) is used to classify an autocomponent of signal and then isolated it into FRFT domain [9-10]. At the end, WD of this isolated component is computed which is free of cross-terms. This technique fails to extract the multi-component signal embedded by AWGN (Additive White Gaussian Noise) [3].

\section{PROBLEM FORMULATION}

In this paper a free cross-term $t-f$ representation of WD is presented, when a multi-component signal is embedded by AWGN. The proposed approach analyze a signal component with noise is check to extract all the signal components through rectified STFT and kurtosis method [12-13]. Image segmentation and SVM used for classifying a signal components and then all signal components filter out by FRFT technique [10-14]. A crisp (free of crossterms) $t$-f representation is obtained by computing WD of the separated signal components.

\subsection{Rectified Short Time Fourier Transform}

STFT is a linear $\mathrm{t}-\mathrm{f}$ representation which allows the frequency description of a varying signal component with respect to the time [12]. A signal which carry information and that information vary w.r.t the time. Such signal presents a meaningful features in frequency domain. However, to represent an instantaneous frequency in frequency domain. A simple extension is carried out called DFT (Discrete Fourier Transform). DFT gives a discrete output in frequency domain. FFT is also a DFT, but the number of samples taken twice a power to accelerate the computation in frequency domain. STFT is a collection of $N$ time FT (Fourier Transforms) computed with a sliding window $\mathrm{h}[\mathrm{k}-\mathrm{m}]$. The advantage of using a window function other than rectangular window is to have a lower side lobes. Lower side lobes lose resolution in frequency 
domain. The plot of magnitude of STFT called spectrogram, which gives signature of signal in both time and frequency domain. Consider a multi-component signal $x(t)$ embedded with AWGN named ass $(t)$. It can be defined as:

$s(t)=x(t)+w(t)$

In discrete form,

$s[n]=x[n]+w[n]$

$\mathrm{S}[\mathrm{m}, \mathrm{n}]=\sum_{\mathrm{k}} \mathrm{s}[\mathrm{k}] \mathrm{h}[\mathrm{k}-\mathrm{m}] \mathrm{e}^{(-\mathrm{j} 222 \pi)}$

$\mathrm{S}[\mathrm{m}, \mathrm{n}]=\mathrm{S}_{\mathrm{r}}(\mathrm{m}, \mathrm{n})+\mathrm{S}_{\mathrm{i}}[\mathrm{m}, \mathrm{n}]$

Where, $\mathrm{k}$ is a number of delays to slide a window, $\mathrm{m}$ and $\mathrm{n}$ are time and frequency indexes and $\mathrm{h}[\mathrm{k}-\mathrm{m}]$ is a window.

The Real and Imaginary part of STFT is a summation of K Gaussian variables. The mean and variance of STFT of $s[n]$ given in Equation (4) for real and imaginary part are:

(a) Mean

$\mathrm{E}\left(\mathrm{S}_{\mathrm{r}}[\mathrm{m}, \mathrm{n}]\right)=\sum_{\mathrm{k}} \mathrm{s}[\mathrm{k}] \mathrm{h}[\mathrm{k}-\mathrm{m}] \cos (-2 \pi \pi \mathrm{n})$

$\mathrm{E}\left(\mathrm{S}_{\mathrm{i}}[\mathrm{m}, \mathrm{n}]\right)=\sum_{\mathrm{k}} \mathrm{s}[\mathrm{k}] \mathrm{h}[\mathrm{k}-\mathrm{m}] \sin (-2 \pi \pi \mathrm{n})$

(b) Variance

$\sigma_{\omega_{\mathrm{r}}}^{2}=\operatorname{Var}\left(\mathrm{S}_{\mathrm{r}}[\mathrm{m}, \mathrm{n}]\right)=\sigma^{2} \sum_{\mathrm{k}} \mathrm{h}[\mathrm{k}-\mathrm{m}]^{2} \cos (-2 \pi \pi \mathrm{n})^{2}$

$\sigma_{\omega_{\mathrm{i}}}^{2}=\operatorname{Var}\left(\mathrm{S}_{\mathrm{i}}[\mathrm{m}, \mathrm{n}]\right)=\sigma^{2} \sum_{\mathrm{k}} \mathrm{h}[\mathrm{k}-\mathrm{m}]^{2} \sin (-2 \pi \pi \mathrm{n})^{2}$

The STFT of an AWGN coefficient do not have a Gaussian distribution. Therefore, a Rectified STFT of a given signal is computed by [12]:

$$
\begin{aligned}
& \mathrm{S}_{\mathrm{r}}[\mathrm{m}, \mathrm{n}]^{\prime}=\frac{\mathrm{S}_{\mathrm{r}}[\mathrm{m}, \mathrm{n}]}{\sqrt{\alpha[\mathrm{m}, \mathrm{n}]}} \\
& \mathrm{S}_{\mathrm{r}}[\mathrm{m}, \mathrm{n}]^{\prime}=\frac{\mathrm{S}_{\mathrm{i}}[\mathrm{m}, \mathrm{n}]}{\sqrt{1-\alpha[\mathrm{m}, \mathrm{n}]}}
\end{aligned}
$$

Where $\alpha[\mathrm{m}, \mathrm{n}]$ is the ratio of variance of real part and sum of variance of real and imaginary part.

$\mathrm{a}[\mathrm{m}, \mathrm{n}]=\operatorname{Var}\left(\left[\mathrm{S}_{\mathrm{r}}[\mathrm{m}, \mathrm{n}]\right) /\left[\operatorname{Var}\left(\left[\mathrm{S}_{\mathrm{r}}[\mathrm{m}, \mathrm{n}]\right)+\operatorname{Var}\left(\left[\mathrm{S}_{\mathrm{i}}[\mathrm{m}, \mathrm{n}]\right)\right]\right.\right.\right.$

$$
\left.\alpha[\mathrm{m}, \mathrm{n}]=\frac{\sigma_{\omega_{\mathrm{r}}}^{2}}{\left(\sigma_{\omega_{\mathrm{r}}}^{2}+\sigma_{\omega_{\mathrm{i}}}^{2}\right.}\right)
$$

\subsection{Support Vector Machine}

The technique used for the classification of signal components in t-f plane is called as SVM [3-14]. In this technique, we insert boundary values between signal components which are segmented in t-f plane. This segmented is represented as $Q$.

$\mathrm{Q}=\left.\left(\mathrm{y}_{\mathrm{i}} \mathrm{a}_{\mathrm{i}}\right)_{\mathrm{a}_{\mathrm{i}} \in\{-1,1\}}^{\mathrm{y}_{\mathrm{i}} \in \mathrm{R}^{2}}\right|_{\mathrm{i}=1} ^{\mathrm{j}}$

Where $y_{j}=2 D$ vector show pixel value related to segmented image in t-f plane, $a_{\mathrm{j}}=-1$ or 1 , showing segmented image to which the point related, Our main emphasis is to find out a line that gives a maximum margin between the points. Mathematically, we can write as having the value of $a_{\mathrm{j}}=1$ and -1 :

S.X $-c=1$

S.X $-c=-1$

\subsection{Fractional Fourier Transform}

FFT is used to compute the free cross-term t-f representation, whereas the classical approach of a signal 
in frequency domain, Fourier Transform is used [10]. If the signal components are very close to each other in $t-f$ plane, then it cannot be filtered out easily. The formulation of FRFT becomes a powerful tool for the analysis of timevarying signal. The closest intact between FT and FRFT, play a major role in signal processing applications, such as filtering, signal detection, image reconstruction, image enhancement and t-f plane etc. [15]. FRFT of a given signal $\mathrm{x}(\mathrm{t})$ is written as:

$X_{\beta}(u)=\int G(\beta, t, u) x(t) d t$

Where

$G(\beta, t, u)=\frac{t^{2}+u^{2} \cos (\beta-2 t u)}{\sin \beta \sqrt{j \sin \beta}} e^{j\left(\frac{\beta}{2}+\pi\right)}$

Where $\beta=n \pi / 2, n=1,2,3, \ldots$, and $G(\beta, t, u)$ is a kernel function. When $\mathrm{n}=1$, FRFT becomes FT, i.e. $\beta=\pi / 2$.

By using Equation (17) each signal component is filteredout in t-f plane obtained by SVM through following procedure:

- $\quad$ Compute the $\alpha$ order FRFT of given signal (i.e. rotate +ve in t-f plane of signal component)

- $\quad$ Filter the signal component through band-pass filter

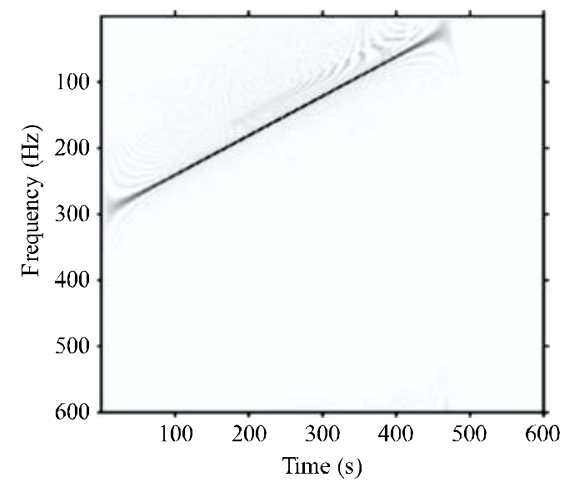

(a)

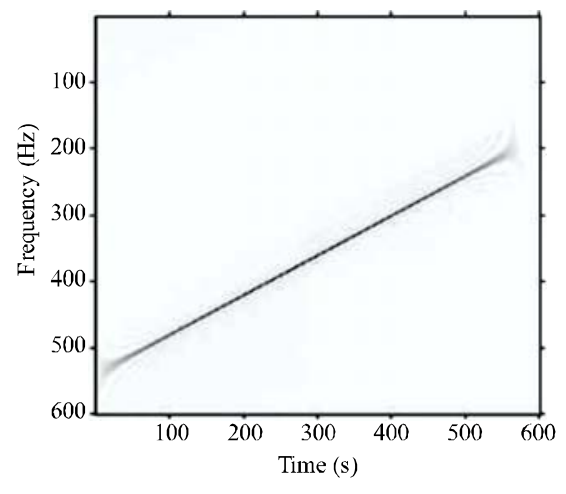

(b)
Again, compute the $\alpha$ order FRFT (i.e. rotate -ve in $t-f$ plane of signal component)

\subsubsection{Wigner Distribution}

WD is used to estimate ideal frequency in $\mathrm{t}-\mathrm{f}$ plane when we deal with mono-component signal and it is suffered from cross-terms when it deals with multi-component signal [16]. WD can be defined in term of FT for instantaneous auto-correlation function as:

$$
\mathrm{W}_{\mathrm{x}}(\mathrm{t}, \mathrm{f})=\int\left[\mathrm{x}\left(\mathrm{t}+\frac{\tau}{2}\right)\right]\left[\mathrm{x}^{*}\left(\mathrm{t}-\frac{\tau}{2}\right)\right] \mathrm{e}^{-\mathrm{j} 2 \pi 2 \pi} \mathrm{d} \tau
$$

Here $\tau$ is the number of delay.

WD is quadratic in nature, therefore cross-terms appears when a signal is composed more than one component. From the following relation, it can be defined as:

$$
\begin{gathered}
\mathrm{W}_{\mathrm{x}}(\mathrm{t}, \mathrm{f})=\sum_{\mathrm{k}=1}^{\mathrm{N}} \mathrm{W}_{\mathrm{x}_{\mathrm{k}} \mathrm{x}_{\mathrm{k}}}(\mathrm{t}, \mathrm{f})+\sum_{\mathrm{i}=1}^{\mathrm{N}} \sum_{\mathrm{j}=1}^{\mathrm{N}} \mathrm{W}_{\mathrm{x}_{\mathrm{i}} \mathrm{x}_{\mathrm{j}}}(\mathrm{t}, \mathrm{f}) \\
\mathrm{i} \neq \mathrm{j}
\end{gathered}
$$

Where

$$
\mathrm{W}_{\mathrm{x}_{\mathrm{k}} \mathrm{x}_{\mathrm{k}}}(\mathrm{t}, \mathrm{f})=\int\left[\mathrm{x}_{\mathrm{k}}\left(\mathrm{t}+\frac{\tau}{2}\right)\right]\left[\mathrm{x}_{\mathrm{k}}^{*}\left(\mathrm{t}-\frac{\tau}{2}\right)\right] \mathrm{e}^{-\mathrm{j} 2 \pi 2 \pi} \mathrm{d} \tau
$$

FIG. 1. FRACTIONAL FILTERING OF LINEAR COMPONENTS 


$$
\mathrm{W}_{\mathrm{x}_{\mathrm{i}} \mathrm{x}_{\mathrm{j}}}(\mathrm{t}, \mathrm{f})=\int\left[\mathrm{x}_{\mathrm{i}}\left(\mathrm{t}+\frac{\tau}{2}\right)\right]\left[\mathrm{x}_{\mathrm{j}}^{*}\left(\mathrm{t}-\frac{\tau}{2}\right)\right] \mathrm{e}^{-\mathrm{j} 2 \pi 2 \pi} \mathrm{d} \tau
$$

For linear frequency modulation, Equation (20) gives optimum energy concentration in t-f plane due to autocomponents. On the other hand, when a signal is nonlinearly frequency modulated then Equation (21) gives unwanted interference terms called cross-terms. Crossterms arise due to non-linear nature of signal [17].Thus for such signal; desired t-f representation is given as:

$$
P(t, \omega)=\sum_{j=1}^{k} S(t, \omega) * W_{s_{j}}(t, \omega)
$$

$\mathrm{P}(\mathrm{t}, \omega)$ is obtained from isolated signal components by computing the $\mathrm{S}^{*} \mathrm{WD}$ of isolated signal components and then added up to individually in t-f plane.

\section{PROPOSED APPROACH}

The main objective of this research is to propose an efficient technique to compute free cross-terms $t-f$ representation of WD, when a multi-component signal is embedded with AWGN. We take a signal of Equation (1) and compute rectified STFT by using Equation (11).

The main steps of the technique are:

- Rectified Short Time Fourier Transform:

- Image segmentation

- Classification of signal Components

- $\quad$ Fractional Filter

- Wigner Distribution of Separated Signal Components

\subsection{Rectified Short Time Fourier Transform}

Noise variance is estimated using rectified STFT coefficients in t-f plane [12-20]. The Noise is overestimated by the presence of signal components. Therefore the Rectified STFT is computed from Equation (11) is given as:

$$
\mathrm{E}\left(\sigma_{\omega}^{2}\right)=\sigma_{\omega}^{2}+\frac{1}{\mathrm{MN}} \sum_{\mathrm{m}, \mathrm{n}}\left|\mathrm{S}_{\mathrm{r}}[\mathrm{m}, \mathrm{n}]\right|^{2}
$$

A threshold value and false alarm probability is computed from Equation (23). Threshold value tells that signalcomponents are not in Gaussian distribution and false alarm probability tells us how much signal involves in noise. It is an iterative process and a threshold value is computed at each number of iterations.

$$
\mathrm{T}_{\sigma_{\omega}^{2}}=\sigma_{\omega}^{2} \ln \left(\mathrm{p}_{\mathrm{fa}}^{-1}\right)
$$

Here $\mathrm{p}^{-1}{ }_{\mathrm{fa}}$ is the False Alarm Probability. There will be two images $I_{1}$ and $I_{2}$ appeared, when threshold is applied. Image $I_{1}$ consist of signal components value that is greater than a certain threshold stored in image $\mathrm{I}_{1}$. Similarly, Image $\mathrm{I}_{2}$ consists of remaining undetected points. Again, Noise variance is re-estimated from image $\mathrm{I}_{2}$ and only those points $\mathrm{I}_{2}$ are classified as a signal component as computed in earlier step for image $\mathrm{I}_{1}$. It is an iterative process and is repeated until all signal components removed from image $I_{2}$. Kurtosis [12-19] is computed from image $I_{2}$ classified as a Noise, where

$$
K_{s}=\frac{E\left(S^{2} S^{* 2}\right)}{E\left(S S^{*}\right)}-2
$$

When Spectral kurtosis value of image $\mathrm{I}_{2}$ approaches zero, it means the remaining undetected points are Noise and its distribution is equal to a GD.

\subsection{Image Segmentation}

Image $I_{1}$ has a non-zero value in $t-f$ plane which indicates that a signal component is present and it has zero value 
in t-f plane where a signal component is not present [19]. Image $_{1}$ is converted into binary image. Mathematically:

$I_{M}[m, n]= \begin{cases}1 & \text { ifI }_{M}(m, n) \geq R \\ 0 & \text { ifI }_{M}(m, n)<R\end{cases}$

Where $\mathrm{R}$ is threshold value.

It is assumed that all signal components having nonoverlap t-f signature. The main emphasis of image segmentation is to decompose signal components from the foreground of $t-f$ image [14]. Therefore, a t-f image is decomposed into number of foreground segment and one background segment. Mathematically, it is expressed as:

$$
\mathrm{I}_{\mathrm{M}}=\cup_{\mathrm{k}=1}^{\mathrm{N}} \mathrm{S}_{\mathrm{k}} \cup \mathrm{I}_{0}
$$

Where, $I_{M}$ is a set of pixels which consist of all foreground and background t-f image $S_{k}$ is a set of pixels representing $\mathrm{S}_{\mathrm{k}}(\mathrm{t})$ in time domain. A signal component is segmented by applying an 8-connectivity criterion [14]. Shown in Fig. 1(e).

According to these criteria, 8-neighborhood of a pixel is defined a location of $(\mathrm{x}, \mathrm{y})$ which contains a set of all pixels value at a following locations:

$$
\prod_{\substack{n=-1 \\ m=-1}}^{1}(x+n, y+m)
$$

The expanded expression of Equation (28), the 8neighborhood of a pixel are given as:

$[(\mathrm{x}-1, \mathrm{y})(\mathrm{x}+1, \mathrm{y})(\mathrm{x}-1, \mathrm{y})(\mathrm{x}+1, \mathrm{y})(\mathrm{x}-1, \mathrm{y}+1)(\mathrm{x}+1, \mathrm{y}-1)(\mathrm{x}, \mathrm{y}-1)(\mathrm{x}, \mathrm{y}-1)]$

\subsection{Signal Component Classification}

SVM is used for the classification of signal components in t-f plane [3].To filter-out the signal components from segmented t-f image, we need to draw a separating boundary between signal components. One line is enough to separate the signal components if two signal components are linearly separable in t-f plane and multiple lines are used to separate the signal components if they are non-linear. It is an iterative process and is repeated till all the segmented regions are separated by lines.

\subsection{Fractional Filter}

FF (Fractional Filter) is employ to filter-out the separated signal components of a segmented signal in t-f plane [2122]. From the slope of line that is drawn to differentiate between two segments, Rotating order of FF is computed and cut-off frequency is projected from the -intercept of separating between two segments. This process is recursive until all the signal components are filtered-out in t-f plane [9-10].

Compute the order of FRFT of signal in t- $f$ plane and rotate it in direction then filter to separate out it in $\mathrm{t}-\mathrm{f}$ plane [7]. Again compute the order of FRFT of signal in t$\mathrm{f}$ plane and rotate in - direction to achieve a separated signal component in t-f plane as shown in Fig. 1(f-h).

\subsection{Wigner Distribution of Separated Signal Components}

WD of separated signal component is computed after filtering signal components in t-f plane by FF [22-23]. Now we have two choices to compute the WD, depends upon the nature of the signal.

- If a signal component is linearly frequency modulated in t-f plane than WD is an optimum choice.

- If a signal component is non-linearly frequency modulated in $\mathrm{t}-\mathrm{f}$ plane than compute a product of rectified STFT(s) and WD. 
Finally, the Resulting isolated components $\mathrm{P}_{\mathrm{Si}}(\mathrm{t}, \omega)$ are added and normalized by the maximum value of WD to obtain a desired $t-f$ representation.

$\mathrm{P}_{\mathrm{S}_{j}}(\mathrm{t}, \omega) \frac{\sum_{\mathrm{j}=1}^{\mathrm{N}} \mathrm{WD}_{\mathrm{S}_{\mathrm{j}}}(\mathrm{t}, \omega) \mathrm{S}_{\mathrm{S}_{\mathrm{j}}}(\mathrm{t}, \omega)}{\mathrm{WD}_{\mathrm{j}}^{\max }(\mathrm{t}, \omega)}$

\section{NUMERICAL SIMULATION}

For analyzing the proposed approach we test the signal and show their respective simulation by using CAD simulator according to the Flow chart shown in Fig. 2.

Example-1: Consider a noisy signal (SNR 2dB) consist of three non-linear signal component defined as:

$\mathrm{S}(\mathrm{t})=0.3 \mathrm{e}^{-\mathrm{j}\left(2 \pi \pi 3^{4}+2 \pi \pi 30\right)}+0.5 \mathrm{e}^{-\mathrm{j}\left(2 \pi \pi 5^{4}+2 \pi \pi 50\right)}+0.7 \mathrm{e}^{-\mathrm{j}\left(2 \pi \pi 5^{4}+2 \pi \pi 70\right)}$

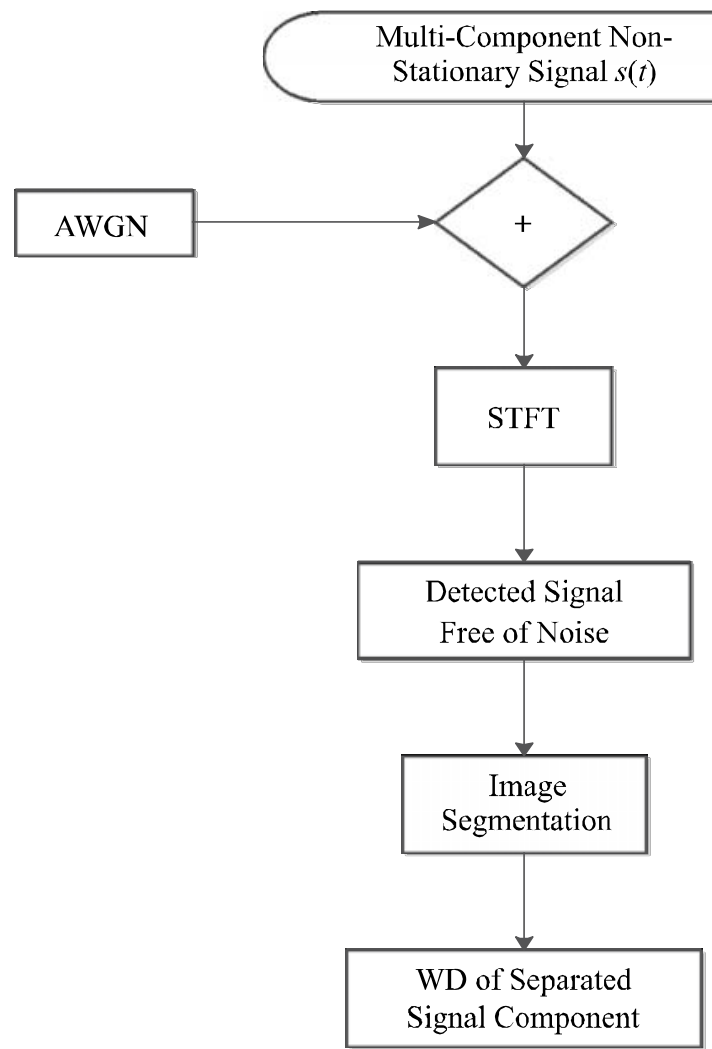

FIG.2: FLOW CHART OF PROPOSED TECHNIQUE
The simulation is taken at sampling frequency of $200 \mathrm{~Hz}$ and time duration is -1.5-1.5 seconds as shown in Fig 3(a). The signal components are corrupted by AWGN at SNR $>2 \mathrm{dBis}$ shown in Fig 3(b). Signal components are detected by applying proposed algorithm of Equation (23) is shown in Fig 3(c). Signal components are segmented by applying 8-connectivety criteria is shown in Fig 3(d). By drawing boundaries between segmented image multiple lines due to non-linear signal components using SVM is shown in Fig. 3(e). Each (Lower, Middle and Upper) signal component is separately simulated by using FF is respectively shown in Fig. 3(f-h). At the end, a crisp representation is obtained by computing WD of all signal components is shown in Fig. 3(i). The comparison of performance measurement of GT, WD and GWT with proposed approach is given Table 1 .

Example-2: In this example, Consider a noisy signal (SNR $1 \mathrm{~dB}$ ) consist of two quadratic component and a Gaussian atom is given as:

$\mathrm{S}(\mathrm{t})=0.9 \mathrm{e}^{-\mathrm{j}\left(2 \pi \pi 3^{3}+2 \pi \pi 55\right)}+0.8 \mathrm{e}^{-\mathrm{j}\left(2 \pi \pi \mathrm{T}^{3}+2 \pi \pi 15\right)}+0.7\left(\mathrm{e}^{-\mathrm{j}(2 \pi \pi 85)}\right) \mathrm{e}^{-15 \mathrm{t}^{2}}$

The simulation is taken at sampling frequency of $200 \mathrm{~Hz}$ and time duration is -1.5 to 1.5 seconds as shown in Fig. 4(a). The signal components are corrupted by AWGN at SNR $>2$ dBis shown in Fig. 4(b). Signal components are detected by applying proposed algorithm of Equation (22) is shown in Fig. 4(c). Signal components are segmented by applying 8-connectivety criteria is shown in Fig. 4(d). By drawing boundaries between segmented images multiple lines due to non-linear signal components using SVM is shown in Fig. 4(e). Each (Lower, Middle and Upper) signal component is separately simulated by using fractional filter is respectively shown in Fig. 4(f-h). At the end, a crisp representation is obtained by computing WD of all signal components is shown in Fig. 4(i). The comparison of performance measurement of GT, WD and GWT with proposed approach is given Table 2. 

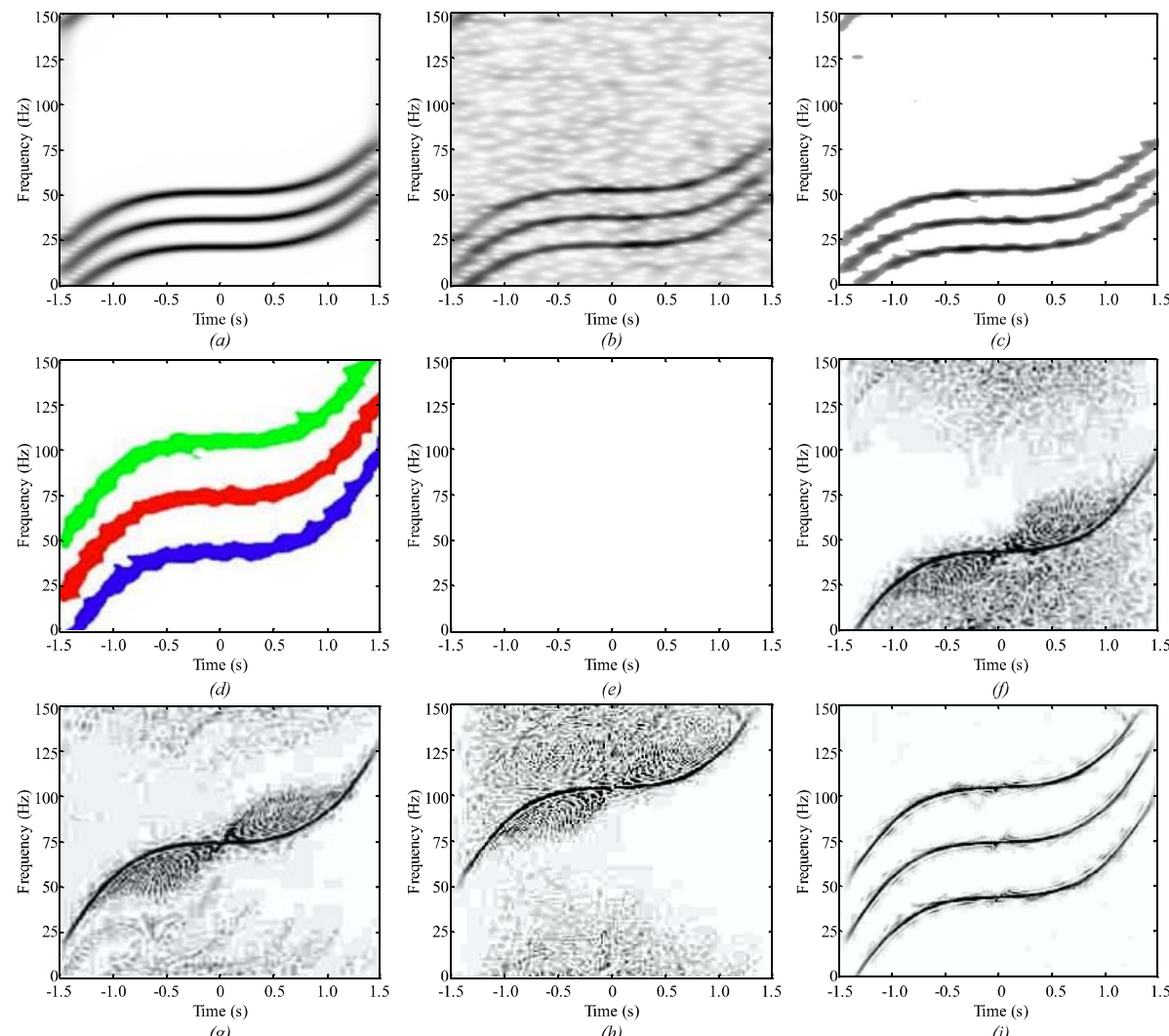

FIG. 3. ANALYSIS OF THREE NON-LINEAR SIGNAL COMPONENTS (A) T-F REPRESENTATION (B) NOISY SIGNAL (C) EXTRACTED SIGNAL FROM NOISE (D) IMAGE SEGMENTATION (E) DRAWING BOUNDARIES USING SVM (F, G, H) FILTERED SIGNAL COMPONENT (I) WIGNER DISTRIBUTION OF ALL SIGNAL COMPONENT.

TABLE 1. PERFORMANCE ANALYSIS (TEST SIGNAL-1)

\begin{tabular}{|c|c|c|c|c|}
\hline T-F Representation & Renyi & Shannon & Ratio of Norms & Ljubisa \\
\hline GT & 15.8943 & 15.7654 & 0.0672 & 1.971 \\
\hline GWT & 12.5431 & 12.6539 & 0.3039 & 0.1768 \\
\hline WD & 13.3417 & 14.8971 & 0.1964 & 3.1789 \\
\hline PAGE & 13.9103 & 15.1054 & 0.0003 & 4.4593 \\
\hline Pseudo WD & 12.9713 & 13.8421 & 0.0007 & 3.6321 \\
\hline ZAM & 14.4007 & 12.0987 & 0.0005 & 2.4539 \\
\hline Proposed Approach & 11.3426 & 13.9875 & 0.2897 & 0.1893 \\
\hline
\end{tabular}

\section{CONCLUSION}

In this paper, we present a novel scheme for computing WD without affecting the quality of auto-terms. In this scheme we use image segmentation and FF technique. An optimized algorithm is proposed for extracting the signal components from noise. A CAD simulator is designed for Numerical simulations of synthetic signals.
In simulation results and the comparison analysis of two examples i.e., non-linear multi-components and non-linear multi-components with a Gaussian atom are simulated. The performance analysis of proposed approach in terms of resolution is better than other t-f representation and also WD of this approach is completely free of cross terms. 

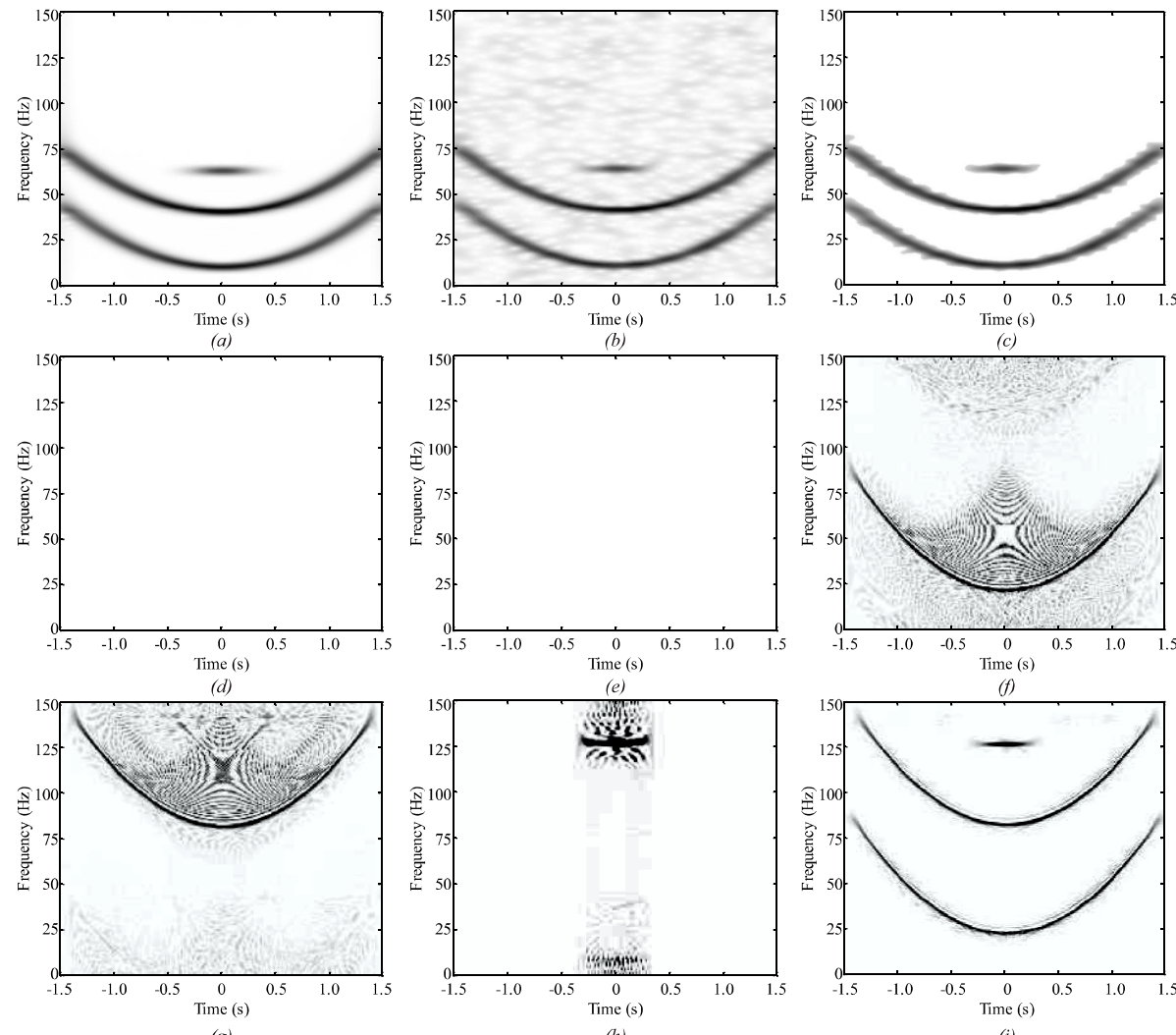

FIG. 4. ANALYSIS OF TWO NON-LINEAR SIGNAL COMPONENT AND GAUSSIAN ATOM(A) T-F REPRESENTATION (B) NOISY SIGNAL (C) EXTRACTED SIGNAL FROM NOISE (D) IMAGE SEGMENTATION (E) DRAWING BOUNDARIES USING SVM (F, G, H) FILTERED SIGNAL COMPONENT (I) WIGNER DISTRIBUTION OF ALL SIGNAL COMPONENT.

TABLE 2. PERFORMANCE ANALYSIS (TEST SIGNAL-2)

\begin{tabular}{|c|c|c|c|c|}
\hline T-F Representation & Renyi & Shannon & Ratio of Norms & Ljubisa \\
\hline GT & 13.3109 & 12.7640 & 0.0497 & 1.1642 \\
\hline GWT & 14.7783 & 14.5639 & 0.02693 & 0.1792 \\
\hline PAGE & 17.8973 & 15.8973 & 0.08932 & 0.7981 \\
\hline Pseudo WD & 16.4539 & 15.2238 & 0.0809 & 1.1376 \\
\hline ZAM & 15.9982 & 14.9781 & 0.3917 & 2.1943 \\
\hline WD & 17.0982 & 16.4301 & 0.2219 & 5.002 \\
\hline Proposed Approach & 13.1830 & 12.5478 & 0.2816 & 0.2174 \\
\hline
\end{tabular}

\section{NOMENCLATURE}

$\mathrm{t}-\mathrm{f} \quad$ Time-Frequency

WT Wavelet Transform

WD Wigner Distribution

CAD Computer Aided Design

FT Fourier Transform

SNR Signal to Noise Ratio

STFT Short Time Fourier Transform
Db Deci Bell

FRFT Fractional Fourier Transform

GWT Gabor Wigner Transform

GT Gabor Transform

SVM Support Vector Machine

AWGN Additive White Gaussian Noise

CTS Cross-Terms Suppression

Mehran University Research Journal of Engineering \& Technology, Volume 36, No. 2, April, 2017 [p-ISSN: 0254-7821, e-ISSN: 2413-7219] 


\section{ACKNOWLEDGEMENTS}

Authors would like to express my sincere gratitude to my anonymous reviewers/expert, for their useful comments/ suggestions and feedback.

\section{REFERENCES}

[1] Boashash, B., "Time-Frequency Signal Analysis and Processing", Prentice-Hall, Upper Saddle River, NJ, USA, 2003.

[2] Cohen, L., "Time-Frequency Distributions -A Review", IEEE Proceedings, Volume 77, 941-981, 1989.

[3] Khan, N.A., Taj, I.A., Jaffri, N., and Ijaz, S., "CrossTerm Elimination in Wigner Distribution Based on 2D Signal Processing Techniques", Signal Processing, Advances in Fractional Signals and Systems, Volume 91, No. 3, pp. 590-599, 2011.

Hlawatsch, F., Manickam, T.G., Urbanke, R.L., and Jones, W., "Smoothed Pseudo-Wigner Distribution, ChoiWilliams Distribution, and Cone-Kernel Representation: Ambiguity-Domain Analysis and Experimental Comparison", Signal Processing, Volume 43, No. 2, 1995.

Boashash, B., Khan, N.A., and Ben-Jaeur, T., "TimeFrequency Features for Pattern Recognition using HighResolution TFDs: A Tutorial Review”, Digital Signal Processing, Volume 40, pp. 1-30, 2015.

[6] Ajab, M., Taj, I.A.N., and Khan, A.N., "Comparative Analysis of Variants of GWT for Cross-Terms Reduction", Metrological Measurement System, Volume 19, No. 3, pp. 499-508, 2012.

Shafi, I., Ahmad, J., Shah S.I., and Kashif, F.M., "Techniques to Obtain Good Resolution and Concentrated Time-Frequency Distribution: A Review”, EURASIP Journal on Advances in Signal Processing, Article ID, [doi: 10.1155/2009/673539], 2009.

Ajab, M., Taj, I.A., Shafi, I., and Stankovic, S., "A New form of Gabor Wigner Transform by Adaptive Thresholding in Gabor Transform and Wigner Distribution and the Power of Signal Synthesis Techniques to Enhance the Strengths of GWT", Metrological Measurement System, Volume XX, No. 1, pp. 99-106, 2013.

[9] Qazi, S., Georgakis, A., L.K., and Stergioulas, M.S., "Interference Suppression in the Wigner Distribution using Fractional Fourier Transformation and Signal Synthesis", IEEE Transaction in Signal Processing, Volume No. 55, pp. 3150-3154, 2007.
Saxena, R., and Sing, K., "Fractional Fourier Transforms A Novel Tool for Signal Processing", Journal of Indian Institute of Science, Volume No. 85, pp. 11-26, 2005.

[11] Stankovic, L., Milos, D., and Thayannathan, T., "TimeFrequency Signal Analysis with Applications", Artech House, 2013.

[12] Millioz, F., and Martin, N., "Circularity of the STFT and Spectral Kurtosis for Time-Frequency Segmentation in Gaussian Environment", IEEE Transactions in Signal Processing, Volume 59, No. 1, 2011.

[13] Millioz, F., Huillery, J., and Martin, N., "Short Time Fourier Transform Probability Distribution for TimeFrequency Segmentation", Proceedings of IEEE Conference on Acoustics, Speech and Signal Processing, No.3, 2006.

[14] Acharya, T., and Ray, A., "Image Processing-Principles and Applications", Wiley-Interscience, Hoboken, NJ, Chapter-12, 2005.

[15] Sejdic, E., Igor, D., and Jin, J., "Time-Frequency Feature Representation using Energy concentration: An Overview of Recent Advances", Digital Signal Processing, Volume 19, No. 1, pp. 153-183, 2009.

[16] Hlawatsch, F., and Flandrin, P., "The Interference Structure of the Wigner Distribution and Related TimeFrequency Signal Representations in the Wigner Distribution", Theory and Applications in Signal Processing, Elsevier, Amsterdam, Netherlands, pp. 59133, 1997.

[17] Pei, S.C., and Ding, J.J., "Relations between Gabor transform and Fractional Fourier Transform and their Applications for Signal Processing”, IEEE Transactions on Signal Processing, Volume 55, No.10, pp. 4839-4850, 2007.

[18] Hory, C., Martin, N., and Chehikian, A., "Spectrogram Segmentation by means of Statistical features of NonStationary Signal Interpretation", IEEE Transaction on Signal Processing, Volume 50 No.12, pp. 2915-2925, 2002.

[19] Hsu, C.W., Chang, C.C., and Lin, C.J., "A Practical Guide to Support Vector Classification", Technical Report, Department of Computer Science and Information Engineering, National Taiwan University Taipei, 106, Taiwan, 2003.

[20] Picinbono, B., "On Circularity", IEEE Transactions in Signal Processing, Volume 42 No. 12, pp. 3473-3482, December, 1994.

[21] Kutay, M.A., Ozakatas, H.M., Arikan, O., and Onural, L., "Optimal Filter in Fractional Fourier Domains", IEEE Transaction on Signal Processing, Volume 45, No. 5, pp. 1129-1143, 1997.

[22] Stankovic, L.J., Alieva, T., and Bastiaans, M.J., "TimeFrequency Signal Analysis Based on the Windowed Fractional Fourier Transform", Signal Processing, Volume 83, No. 11, pp. 2459-2468, 2003.

[23] Mann, S., and Haykin, S.S., "The Chirplet Transform”, A Generalization of Gabor's Logon Transform, Calgary, Canada, 1991. 